\title{
The oxygen desaturation measured by optical sensor at finger- tip but not at the arm is diminished by continuous positive airway pressure in obstructive sleep apnea
}

\author{
Zhongxing Zhang ${ }^{1,2, *}$, Ming Qi ${ }^{1}$, Gordana Hügli ${ }^{1}$ and Ramin Khatami ${ }^{1,2,3}$ \\ 1 Center for Sleep Medicine, Sleep Research and Epileptology, Clinic Barmelweid AG, Barmelweid, Switzer- \\ land; zhongxing.zhang@barmelweid.ch ; ming.qi@barmelweid.ch ; gordana.huegli@barmelweid.ch ; \\ ramin.khatami@barmelweid.ch \\ 2 Barmelweid Academy, Clinic Barmelweid AG, Barmelweid, Switzerland; zhongxing.zhang@barmelweid.ch ; \\ ramin.khatami@barmelweid.ch \\ 3 Department of Neurology, Inselspital, Bern University Hospital and University of Bern, Bern, Switzerland; \\ ramin.khatami@barmelweid.ch \\ * Correspondence: zhongxing.zhang@barmelweid.ch
}

\begin{abstract}
Obstructive sleep apnea (OSA) is a common sleep disorder, and continuous positive airways pressure (CPAP) is the most effective treatment. Poor adherence is one of the major challenges in CPAP therapy. The recent boom of wearable optical sensors measuring oxygen saturation makes the at-home multiple-night CPAP titrations possible, which may essentially improve the adherence of CPAP therapy by optimizing its pressure in a real-life setting economically. We tested whether the oxygen desaturations (OD) measured in the arm muscle (arm_OD) by gold-standard frequency-domain multi-distance near-infrared spectroscopy (FDMD-NIRS) changes with titrated CPAP pressures in OSA patients together with polysomnography. We found that the arm_OD (2.08 $\pm 1.23 \%$, mean \pm standard deviation) was significantly smaller (P-value $<0.0001$ ) than the fingertip OD (finger_OD) $(4.46 \pm 2.37 \%)$ measured by polysomnography pulse oximeter. Linear mixed-effects models suggested that CPAP pressure was a significant predictor for finger_OD but not for arm_OD. Since FDMD-NIRS measures a mixture of arterial and venous OD, whereas fingertip pulse oximeter measures arterial OD, our results of no association between arm_OD and finger_OD indicate that the arm_OD mainly represented venous desaturation. Arm_OD measured by near-infrared optical sensors may be not a suitable indicator of the effectiveness of CPAP titration.
\end{abstract}

Keywords: Obstructive sleep apnea; Continuous positive airways pressure therapy; Near-infrared spectroscopy; Oxygen desaturation; Arm; Pulse oximeter

\section{Introduction}

Obstructive sleep apnea (OSA) is the most prevalent respiratory sleep disorder occurring in $9-38 \%$ of the general population [1], and it is a high risk factor for cardio-/cerebrovascular diseases [2,3]. Continuous positive airways pressure (CPAP) or automatic positive airway pressure (APAP) therapy is currently the most effective treatment for OSA [4,5]. However, there are still many challenges in OSA diagnosis and treatment, particularly simple low-cost new diagnostic technologies that can be easily used by the patients for home recordings, are urgently needed [6,7]. This urgency is due to observations that 1 ) the majority of suspected OSA patients remain undiagnosed $[8,9]$ in many countries due to the lack of sleep laboratories/specialists and the high-cost of in-lab polysomnography (PSG) diagnosis, and 2) long-term home recordings employing 
these easy-to-use technologies will be helpful to longitudinally monitor the CPAP treatment effects and adherence or even to select patients who will benefit most from treatment (i.e., precision or personalized medicine) $[6,10]$.

Recently, low-cost wearable devices, such as smartwatches (e.g., the new products from Fitbit [11], Garmin [12], Huami [13], Huawei [14]) and armbands (e.g., Humon $[15,16]$, Moxy [17,18], Artinis [19] and Biofourmis [20]) have implemented the function of measuring peripheral capillary $(\mathrm{SpO} 2)$ or muscle tissue $(\mathrm{StO} 2)$ oxygen saturation at the arm or wrist using near-infrared light, making them possible alternatives for at-home OSA measurement. Unlike the in-lab PSG fingertip pulse oximetry, in which the detector measures the light transmitted through the fingertip (i.e., the transmission photoplethysmography, T-PPG), these wearable devices assess the $\mathrm{SpO} 2$ or $\mathrm{StO} 2$ by measuring the changes in the backscattering light from the wrist or arm based on the modified Beer-Lambert law (MBLL) [21-24]. That is, their light sources that are usually two or more near-infrared light wavelengths mainly absorbed by oxygenated hemoglobin $(\mathrm{HbO} 2)$ and deoxygenated hemoglobin $(\mathrm{HHb})$ in biological tissues, and detectors are placed on the same side of the measured tissues, because the wrist and arm are too thick to be penetrated through by the light. While the wrist wearable devices mainly use reflectance photoplethysmography (R-PPG) or pulse oximetry [24-26], the armbands are mainly based on simple continuous wave near-infrared spectroscopy (CW-NIRS) methods [16,18].

Measuring OSA by these low-cost wearable devices is unfortunately still not viable, because none of them has been licensed or certified as a medical diagnostic device by the United States Food and Drug Administration or CE marking [27]. In our recent study [28] we compared event-by-event oxygen desaturation (OD) at the fingertip (finger_OD) measured by gold-standard in-lab PSG fingertip T-PPG with the OD at arm (arm_OD) measured by gold-standard frequency-domain multi-distance near-infrared spectroscopy (FDMD-NIRS) in OSA patients during naïve sleep and during CPAP titrations. Our results of Bland-Altman plots gave poor agreements between finger_OD and arm_OD in sleep hypopneas, suggesting that the absolute value of arm_OD is not suitable to define sleep hypopneas according to the standard clinical criterion of $\geq 3 \%$ OD drop. It could lead to large false negative results in measuring OSA events, thus underestimating the Apnea-Hypopnea index (AHI) compared to in-lab PSG.

However, whether the qualitative changes in arm_OD can indicate the CPAP titration effectiveness remains unknown. The CPAP/APAP machine itself can measure AHI, although its accuracy has been criticized [29-31]. Two major challenges in CPAP/APAP treatment are poor adherence to treatment [32] and persisting hypoxemia and daytime syndromes despite therapy in some patients, for whom oxygen supplementation may be needed [6]. Logically, 'A does not equal to $B$ ' does not necessary mean that 'changes in A is not associated with changes in $\mathrm{B}^{\prime}$. For example, obesity is a risk of OSA. Large BMI cannot be used to replace large AHI to define OSA, but the decrease in BMI is associated with decrease in AHI [33]. If the changes in arm_OD can be associated with changes in CPAP/APAP pressures, then measuring changes in arm_OD with a wearable device at home could be helpful to better understand those patients, e.g., to identify subgroups with/without improvement in OD 
when CPAP/APAP pressure changes. It could also make at-home multiple night CPAP titrations possible, i.e., to find the optimal CPAP pressure that can at best restore the respiratory events and arm_OD, which may essentially improve the adherence of CPAP therapy by optimizing its pressures in a real-life setting economically.

Therefore, in this study we quantify the dynamic changes in arm_OD in OSA events under titrated CPAP pressures using regression analysis. We use the same dataset that has been recently published in [28], considering its major advantages, which include: 1) the CPAP pressures were well-controlled stepwise and increased hourly during the titrations; 2) the simultaneously measured finger_OD can serve as a control for CPAP titration effectiveness, i.e., an effective titration procedure should restore stepwise the fingertip SpO2 desaturation; 3) the comparisons between finger_OD (i.e., arterial desaturation) and arm_OD (i.e., the mixture of arterial and venous desaturation $[34,35])$ may provide new insights into the peripheral desaturation in response to OSA events during CPAP therapy. We hypothesized that the changes in arm_OD may be associated with changes in CPAP pressures, considering that our stepwise incremental CPAP titration gradually opened the upper airway to increase oxygen supply.

\section{Materials and Methods}

Thirty newly diagnosed OSA patients (age [mean \pm standard deviation, SD]: $54.2 \pm$ 13.8 years, interquartile range [IQR] $42-65$ years; male: $n=27$; body mass index [BMI]: $35.9 \pm 7.5 \mathrm{~kg} / \mathrm{m}^{2}$, IQR $31.8-42.0 \mathrm{~kg} / \mathrm{m}^{2}$; AHI: $53.4 \pm 24.7$ per hour, IQR 32-71 per hour) participated in this study. Patients with unstable coronary or cerebral artery disease, severe arterial hypertension or hypotension, respiratory diseases or a history of a sleep-related accident were excluded from this study. This study was approved by the local ethical commission of Northwest Switzerland and was in compliance with the Declaration of Helsinki. Written informed consent was obtained from all patients prior to their participation.

Patients underwent incremental stepwise CPAP (AirSense ${ }^{\mathrm{TM}} 10$, ResMed) titration combined with video-PSG and FDMD-NIRS recordings in one nocturnal sleep episode. This sleep episode consisted of 1-h of baseline sleep without CPAP followed by incremental stepwise titration of $1-\mathrm{cmH} 2 \mathrm{O}$ pressure per-hour starting from 5-8 $\mathrm{cmH} 2 \mathrm{O}$ depending on the individuals. Video-PSG (Embla RemLogic, Natus Medical Incorporated, Tonawanda, NY, USA) is a comprehensive recording of physiological signals during sleep, including electroencephalography, electrooculogram, electromyogram, electrocardiogram, breathing functions, heart rate (HR), peripheral fingertip SpO2 and movement during sleep. Two experienced sleep technologists independently scored the sleep stages, respiratory events (sleep apneas and hypopneas), and motion artifacts in 30-s epochs according to the 2017 American Academy of Sleep Medicine manual [36]. The discrepancy between these two technologists was resolved by discussion or recommendation by an experienced neurophysiologist.

FDMD-NIRS (Imagent, ISS, Champaign IL, USA) measurements were conducted over the middle of the left biceps muscle. Imagent is currently the only commercial benchtop FDMD-NIRS device [37-39] and has been CE approved for research. The robustness, precision and accuracy of measuring hemodynamics of the Imagent system 
have been well validated in different physical blood-lipid models [38,40,41] and in vivo studies [42-45]. It has been used as a gold-standard reference measurement of $\mathrm{StO} 2$ for the validations or calibrations of wearable CW-NIRS armbands [16], and portable CW-NIRS oximeters including those have received FDA clearance [41,46]. Its light emitters, four laser diodes at 690-nm wavelength and four laser diodes at 830-nm wavelength are coupled into four light sources and are high frequency modulated at 110 MHz. The light can penetrate into the measured tissues with a depth of several centimeters when the four light sources are aligned and placed at $2 \mathrm{~cm}, 2.5 \mathrm{~cm}, 3 \mathrm{~cm}$ and $3.5 \mathrm{~cm}$ from an optical fiber bundle connected to the photomultiplier tube detector. The sampling rate of FDMD-NIRS recording was set as $5.2 \mathrm{~Hz}$. The Imagent system was calibrated on an optical phantom block to exclude the uncertainty of measurements due to machine errors before each recording. The raw measured NIRS data were subjected to a low-pass $(<0.08 \mathrm{~Hz})$ zero-phase filter designed using Hanning window to remove the physiological noises including heart rate, respiratory noise and spontaneous slow hemodynamic oscillations $[28,47,48]$. The filtered data were then smoothed using the robust locally weighted scatter plot smoothing method [47,49].

The data analysis procedure was shown in Fig.1. After a standard PSG scoring, per-hour AHI under each CPAP pressure was calculated, i.e., the number of event was divided by the sleep time under each CPAP pressure per-hour in the titration protocol. Obstructive apneas $(n=29)$ and hypopneas $(n=31)$ were excluded from analysis if their SpO2 desaturations were larger than $15 \%$ to exclude outliers and potentially unreliable measurements caused by instrument errors $[26,28]$. In each patient all the events under a specific CPAP pressure were also excluded if the corresponding sleep duration under that pressure was shorter than 20 minutes, to exclude the unreliable calculation of the per-hour AHI, e.g., if the sleep duration under some CPAP pressure was just a few minutes while the patient had a number of apneas/hypopneas, the calculated per-hour AHI can be extremely large but its value was unreliable because of short sleep time. Then linear mixed-effects model (LMM) with a random intercept by patients was used to predict the arm_OD and finger_OD caused by the respiratory events, respectively. Explanatory variables were demographic variables (i.e., age, sex, BMI, AHI of the diagnostic night measured by PSG) and parameters that can be measured by CPAP machine or wearable devices, i.e., types of respiratory events, durations of event, sleep stages, mean HR during the events, per-hour AHI under each pressure, and CPAP pressures. Stepwise regression using backward elimination was performed to automatically select the best predictors. Then the final LMM models were built using these best predictors. We reported both the conditional $R^{2}[50]$ and $\Omega^{2}$ [51] to assess the goodness of fit of our final selected models. 


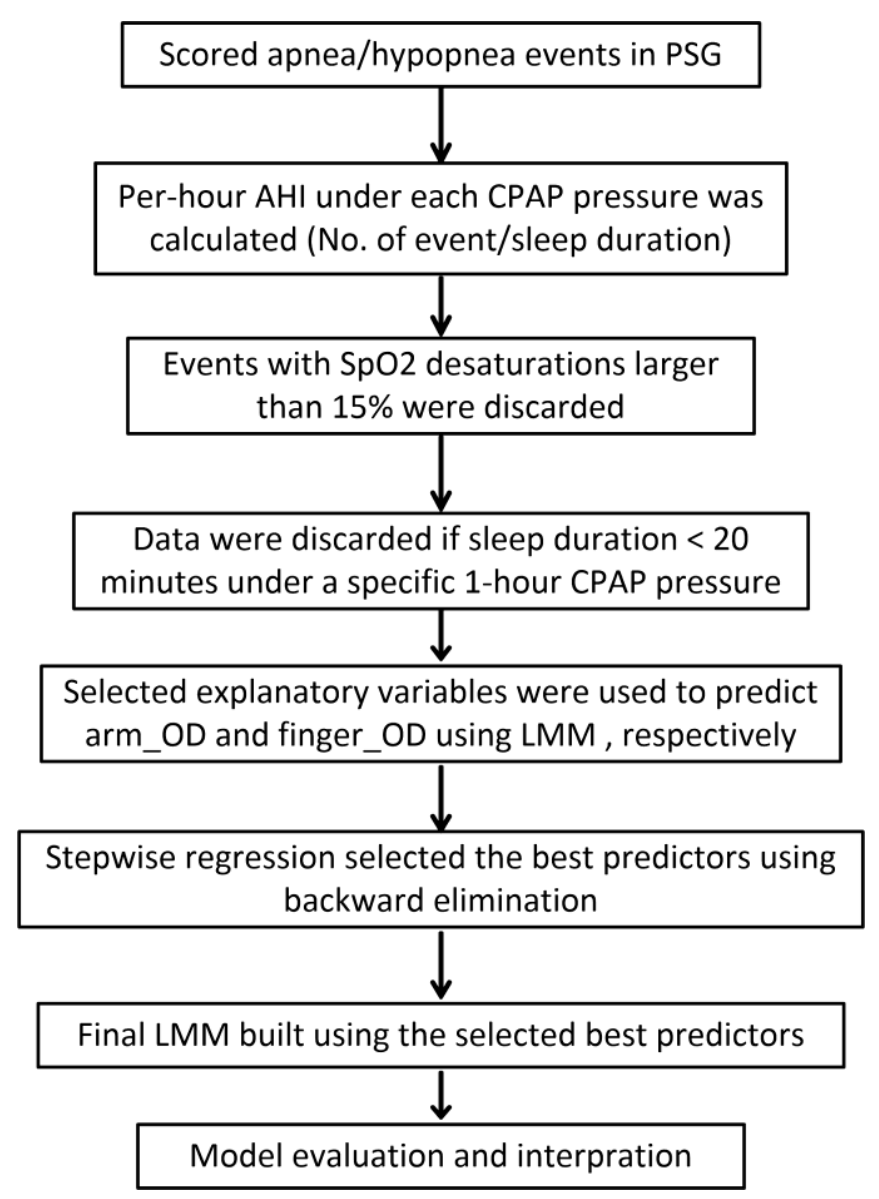

Figure 1. The flowchart of the data analysis process.

Data were expressed as the mean \pm SD unless otherwise indicated. The pre-processing of FDMD-NIRS signals were carried out in MATLAB (The Math-Works, Inc., Natick, MA, USA). All statistical analyses were performed using $\mathrm{R}$ (version 3.2.4). The LMM models were done using the R package lme4 (function lmer) and stepwise regressions were done using the $\mathrm{R}$ package lmerTest (function step).

\section{Results}

In total, 505 obstructive apneas and 2185 hypopneas events were analyzed. The median of the number of events acquired from our patients was 75 with IQR between 59 and 110. Fig.2 illustrates typical changes in fingertip $\mathrm{SpO} 2$ and arm $\mathrm{StO} 2$ desaturations in OSA events. ODs triggered by sleep apneas occurred in both $\mathrm{SpO} 2$ and $\mathrm{StO} 2$, although the baseline $\mathrm{StO} 2$ values (mostly between $60-70 \%$ ) were smaller than $\mathrm{SpO} 2$ (mostly above $90 \%$ ) because $\mathrm{StO} 2$ were from both venous and arterial blood. The mean arm_OD $(2.08 \pm 1.23 \%)$ was significantly smaller (paired t-test, P-value $<0.0001)$ than the mean finger_OD $(4.46 \pm 2.37 \%)$. There was no correlation between the degrees of arm_OD and finger_OD, indicated by the Pearson's correlation coefficient of 0.08 (P-value $<0.0001$ ). 

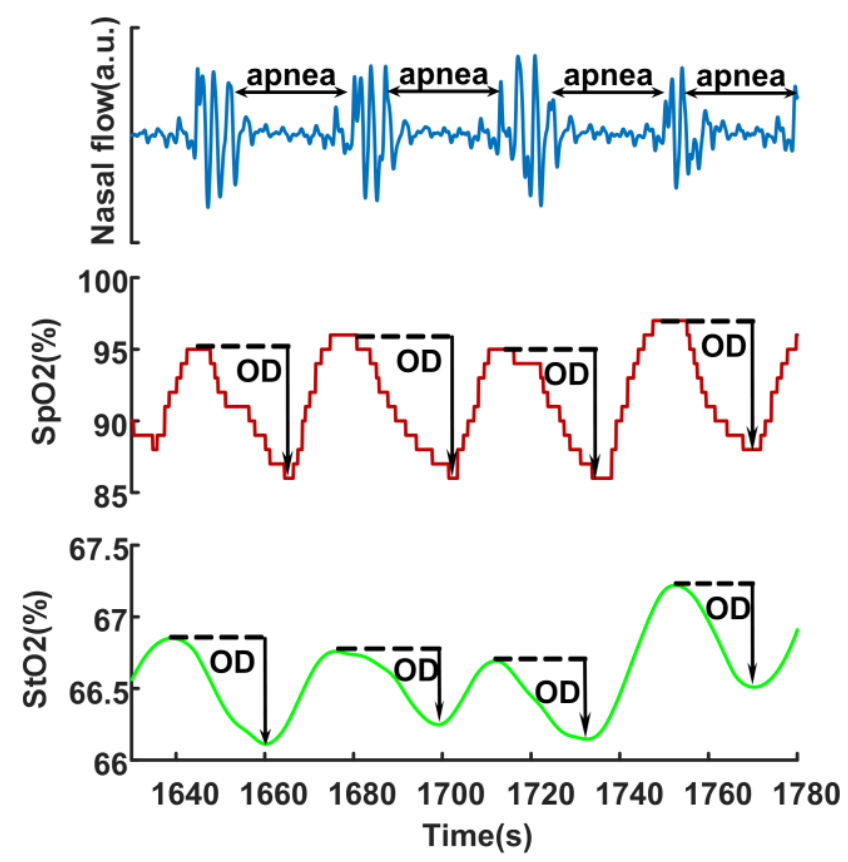

Figure 2. Typical fingertip SpO2 desaturation and arm StO2 desaturation during apneas. The arrow indicates the degree of oxygen desaturation (OD). SpO2 is measured at PSG fingertip by transmission photoplethysmography and $\mathrm{StO} 2$ is measured at biceps muscle by FDMD-NIRS.

The results of the final LMMs predicting arm_OD and finger_OD selected by stepwise regressions are shown in Tables 1 and 2, respectively. The conditional $R^{2}$ and $\Omega^{2}$ of the model for arm_OD were 0.66 and 0.69 , respectively. These two values of the model for finger_OD were 0.51 and 0.49 , respectively. CPAP pressure was a significant predictor for the finger_OD (i.e., the increase of 1 unit pressure was associated with $0.12 \%$ less decrease in fingertip oxygen desaturation) but not for arm_OD.

Table 1. The results of the linear mixed-effects model predicting the degree of oxygen desaturation measured at arm muscle.

\begin{tabular}{lllll}
\hline & Estimate & $\mathbf{9 5 \%} \mathbf{C I}$ & t-value & P-value \\
& $\mathbf{( 1 0 ^ { - 2 } )}$ & $\mathbf{( 1 0 ^ { - 2 } )}$ & & \\
\hline $\begin{array}{l}\text { Duration of } \\
\text { event }\end{array}$ & 2.33 & {$[2.00,2.66]$} & 13.89 & $<0.0001$ \\
$\begin{array}{l}\text { Mean HR within } \\
\text { events }\end{array}$ & -1.49 & {$[-2.14,-0.84]$} & -4.50 & $<0.0001$ \\
per-hour AHI & 0.12 & {$[0.025,0.22]$} & 2.47 & 0.014 \\
\hline
\end{tabular}

CI: confidence interval. HR: heart rate. per-hour AHI is the number of apnea/hypopnea events divided by the sleep time under each CPAP pressure per-hour. 
Table 2. The results of the linear mixed-effects model predicting the degree of oxygen desaturation measured at fingertip.

\begin{tabular}{lllll}
\hline & Estimate & $\mathbf{9 5 \%} \mathbf{C I}$ & & \\
& $\mathbf{( 1 0 ^ { - 2 }}$ & $\mathbf{( 1 0 ^ { - 2 } )}$ & & \\
& & & & \\
\hline Duration of event & 7.83 & {$[7.01,8.66]$} & 18.68 & $<0.0001$ \\
CPAP pressures & -12.12 & {$[-13.93,-10.31]$} & -13.10 & $<0.0001$ \\
Hypopnea-Apnea & -115.6 & {$[-134.9,-96.3]$} & -11.71 & $<0.0001$ \\
per-hour AHI & 1.42 & {$[1.14,1.70]$} & 9.87 & $<0.0001$ \\
Sleep stages & & & & \\
Deep sleep-light sleep & -68.82 & {$[-89.56,-48.08]$} & -6.51 & $<0.0001$ \\
REM sleep-light sleep & -56.41 & {$[-82.13,-30.69]$} & -4.30 & $<0.0001$ \\
AHI of diagnostic night & 1.93 & {$[0.35,3.50]$} & 2.39 & 0.024 \\
\hline
\end{tabular}

CI: confidence interval. CPAP: continuous positive airway pressure. REM: rapid eye movement. per-hour AHI is the number of apnea/hypopnea events divided by the sleep time under each CPAP pressure per-hour. Hypopnea-Apnea means the change in apnea is the reference for the change in hypopnea in this model, i.e., the changes in hypopnea minus the changes in apnea. Light sleep (stage N1 and N2) is the reference for deep sleep (stage N3) and REM sleep.

It could be possible that the normalized changes rather than the raw values of arm_OD were associated with CPAP pressures, since the StO2 baseline level (68.6 $\pm 6.4 \%)$ before the desaturations was obviously much smaller than that of $\mathrm{SpO} 2$ (usually above 90\%). We therefore normalized the arm_OD to its baseline and repeated the LMM analysis. The CPAP pressure was still automatically excluded from the final selected model in stepwise regression, in which the final selected predictors were the same as shown in Table 1, i.e., the duration of events (estimated coefficient 0.033, P-value $<0.0001$ ), mean HR within events (estimated coefficient -0.03 , P-value $<0.0001$ ), per-hour AHI (estimated coefficient 0.0028, P-value $=0.00058$ ).

\section{Discussion}

In this study we test whether oxygen desaturations measured in the arm muscle change with CPAP pressures during CPAP titration in patients with OSA. In contrast to our recent study testing the agreement between arm and fingertip oxygen desaturations in sleep hypopneas using Bland-Altman plots [28], here we use linear regression (i.e., LMM) to study the association between CPAP pressures and oxygen desaturations at arm and fingertip. Contrary to our hypothesis, we only found association between CPAP pressures and the oxygen desaturations at the fingertip but not in the arm muscle. Our negative results suggest that muscular oxygen desaturation may be not a suitable 
indicator of the effectiveness of CPAP titration. Thus, the usefulness of wearable devices measuring arm $\mathrm{StO} 2$ in CPAP therapy is questionable.

Only fingertip $\mathrm{SpO} 2$ but not the arm $\mathrm{StO} 2$ reflects the reduction of desaturations during CPAP titration, probably because venous blood contributing to $\mathrm{StO} 2$ reduces the sensitivity of $\mathrm{StO} 2$ in response to CPAP pressures compared to SpO2. The NIRS StO2 is the proportion of $\mathrm{HbO} 2$ in the measured biological tissues including arterial, capillary and venous compartments. It can be expressed as:

$$
\mathrm{StO} 2=a \times \mathrm{SaO} 2+b \times \mathrm{SvO} 2
$$

where $\mathrm{SaO} 2$ and $\mathrm{SvO} 2$ are the arterial and venous oxygen saturation [34,35]. $\mathrm{SaO} 2$ is approximately equal to $\mathrm{SpO} 2$ as they are both arterial oxygen saturation (usually close to $100 \%)$. SvO2 is usually $65-75 \%$ [52]. The ratio of coefficients $a / b$ is the arterial-to-venous volume ratio (AVR), and $a+b=1$. Most commercially available NIRS oximeters including the FDA-certificated medical devices usually fix the AVR as either $25 \% / 75 \%$ or $30 \% / 70 \%$ [53-63] but never validate them in OSA. If we assume that the fixed AVR model was valid in OSA, we could expect that arm_OD highly correlates with finger_OD considering their mathematical relationship, e.g., $1 \%$ decrease in fingertip arterial $\mathrm{SpO} 2$ may correspond to $0.25 \%$ or $0.3 \%$ decrease in arm StO2 because only a percent $(25 \%$ or $30 \%$ ) of the $1 \% \mathrm{SpO} 2$ desaturation can contribute to $\mathrm{StO} 2$ desaturation according to Formula (1). Thus, similar as the results of finger_OD in response to CPAP titration shown in Table 2, arm_OD should also decrease stepwise with increasing CPAP pressures. However, the lack of correlation between the changes in finger_OD and arm_OD and the missing association between CPAP pressures and arm_OD contradict this assumption, suggesting the fixed AVR model is unlikely to be valid in OSA. In fact OSA event is actually associated with increased vasoconstriction in peripheral limb arteries and arterioles [64,65], which suggests the AVR in the arm muscle is hardly constant during OSA. Most likely the coefficient $a$ decreases while coefficient $b$ increases in OSA because: 1) $a+b=1$ thus an increase in one coefficient must be associated with a decrease in the other one, and arterial vessels have stronger capacity to constrict than venous vessels; 2) it is known that blood pressure, $\mathrm{HR}$, left ventricular stroke volume and cardiac preload all decrease during apnea/hypopnea events [47,64,66-68], indicating more blood may be held in the venous vascular bed. Therefore, the contribution of venous blood to $\mathrm{StO} 2$ increases while arterial blood contribution decreases during OSA events, leading to a reduction in the sensitivity of $\mathrm{StO} 2$ in response to $\mathrm{SpO} 2$ changes. This interpretation also fits our results that arm_OD has a negative association with the mean HR during the events (Table 1), i.e., higher HR may indicate less vasoconstriction (i.e., relative larger $a$ ) and relatively more arterial blood supply to the muscle tissues, ergo arm_OD is smaller.

To the best of our knowledge, NIRS has not been used to measure the peripheral hemodynamics (i.e., muscular hemodynamics) in OSA. Our results of no correlation between finger_OD and arm_OD and the aforementioned increased venous contribution to $\mathrm{StO} 2$ during OSA events indicate that our arm_OD measured by NIRS is likely to mainly represent the OD in venous blood. Our results (Table 1 and Table 2) thus provide new insights into the changes in peripheral oxygen desaturation in OSA events during $\mathrm{CPAP}$, that 1) the arterial but not venous desaturation is more sensitive to changes in 
CPAP pressure; 2) arterial but not venous desaturation depends on the types of events (i.e., apnea causes larger desaturation than hypopnea) and sleep stages (light sleep causes larger desaturation than others); 3) longer events cause larger desaturations in both arterial and venous blood as indicated by longer hypoxia that cause stronger oxygen extraction from both arterial and venous vascular bed.

In our recent study using the same database we reported poor agreements (analyzed by Bland-Altman plots) between arm_OD and finger_OD in sleep hypopneas, and thus the reliability of $\mathrm{AHI}$ measured by $\mathrm{StO} 2$ desaturation using wearable or portable optical sensors based on NIRS technique is questionable [28]. This conclusion could be extended to wearable optical sensors based on R-PPG technique like wrist smartwatches, because they face the same problem of venous blood influence as NIRS [28]. A recent study tested the accuracy of wrist R-PPG smartwatch in measuring SpO2, when $\mathrm{SaO} 2$ measured from blood samples with a co-oximeter changed from $100 \%$ to $70 \%$ [69]. In that study, the Bland-Altman plot gave a broad $95 \%$ lower (i.e., approximate $-4 \%$ ) and upper (i.e., approximate 6-7\%) limits of agreement between smartwatch and co-oximeter measurements, which are similar as those reported in our study [28]. The authors also showed that the $\mathrm{SpO} 2$ measurement error of their smartwatch is 3\% [69]. This accuracy is still too poor to measure sleep hypopneas, because hypopnea is defined as $\geq 3 \%$ OD. Our results do not support the hypothesis that CPAP titration effectiveness may be assessed by measuring peripheral $\mathrm{StO} 2$ desaturation. This conclusion probably can apply to SpO2 measured by smartwatches too.

Our study has several limitations. First, our patients may only represent male patients with severe OSA, because although their age (Shapiro-Wilk normality test: P-value $=0.45$ ) and BMI (Shapiro-Wilk normality test: P-value $=0.66$ ) follow normal distribution, only 3 females were included. Whether our conclusions can be generated to females and patients with moderate OSA needs further studies. Second, correlation and association are not causality. Although we controlled multiple covariates (e.g., HR, sleep stages) in our LMM models, the causal relationships between CPAP pressures and changes in oxygen saturations in fingertip and arm muscle need further studies, e.g., studies with randomized CPAP pressures and/or multi-parameter (e.g., blood flow, endothelium function) measurements in addition to oxygen saturation. The causality analysis can essentially provide new insights into the hemodynamic regulations and consequences of CPAP therapy in OSA [70,71].

\section{Conclusions}

Although the recent boom of wearable optical sensors like smartwatches and armbands offers a possibility of assessing OSA and multiple-night CPAP titrations at home, our negative results should warn the general public and sleep researcher/clinicians to be cautious with these wearable devices until those products are clinically and experimentally validated. Probably more sophisticated algrithms such as machine learning are needed to derive some peripheral parameters than can correctly measure $\mathrm{SpO} 2$ using wearable devices. Our results also suggest that the muscular $\mathrm{StO} 2$ desaturation measured by NIRS may mainly represent venous desaturation in OSA. We suggest that more studies including the gold-standard invasive measurements of $\mathrm{SaO} 2$ 
and $\mathrm{SvO} 2$ together with simultaneous non-invasive NIRS measurement during OSA events are needed, to further test the robustness and reliability of NIRS as a non-invasive tool in measuring $\mathrm{SvO} 2$ in OSA.

Author Contributions: Conceptualization, Zhongxing Zhang and Ramin Khatami; Data curation, Zhongxing Zhang, Ming Qi and Gordana Hügli; Formal analysis, Zhongxing Zhang; Funding acquisition, Zhongxing Zhang and Ramin Khatami; Investigation, Zhongxing Zhang, Ming Qi, Gordana Hügli and Ramin Khatami; Methodology, Zhongxing Zhang; Project administration, Zhongxing Zhang and Ramin Khatami; Resources, Zhongxing Zhang and Ramin Khatami; Software, Zhongxing Zhang; Supervision, Ramin Khatami; Validation, Zhongxing Zhang and Ramin Khatami; Visualization, Zhongxing Zhang; Writing - original draft, Zhongxing Zhang; Writing review \& editing, Zhongxing Zhang and Ramin Khatami. All authors have read and agreed to the published version of the manuscript.

Funding: This work was supported by Clinic Barmelweid Scientific Foundation. The data acquisition work was supported by the Research Fund of the Swiss Lung Association No.2014-22.

Institutional Review Board Statement: The study was conducted according to the guidelines of the Declaration of Helsinki, and approved by the local ethical commission of Northwest Switzerland.

Informed Consent Statement: Written informed consent has been obtained from the patient(s) to publish this paper

Data Availability Statement: The raw data supporting the conclusions of this article are available from the corresponding author on reasonable request.

Acknowledgments: We thank our colleague Ian Clark, PhD in psychology, for English language proof reading our manuscript.

Conflicts of Interest: The authors declare no conflict of interest.

\section{References}

1. Senaratna, C.V.; Perret, J.L.; Lodge, C.J.; Lowe, A.J.; Campbell, B.E.; Matheson, M.C.; Hamilton, G.S.; Dharmage, S.C. Prevalence of obstructive sleep apnea in the general population: A systematic review. Sleep Med Rev 2017, 34, 70-81, doi:S1087-0792(16)30064-8 [pii]10.1016/j.smrv.2016.07.002.

2. Yaggi, H.K.; Concato, J.; Kernan, W.N.; Lichtman, J.H.; Brass, L.M.; Mohsenin, V. Obstructive sleep apnea as a risk factor for stroke and death. New Engl J Med 2005, 353, 2034-2041, doi:Doi 10.1056/Nejmoa043104.

3. Somers, V.K. Sleep--a new cardiovascular frontier. N. Engl. J. Med. 2005, 353, 2070-2073, doi:10.1056/NEJMe058229.

4. Sharma, S.K.; Agrawal, S.; Damodaran, D.; Sreenivas, V.; Kadhiravan, T.; Lakshmy, R.; Jagia, P.; Kumar, A. CPAP for the metabolic syndrome in patients with obstructive sleep apnea. N. Engl. J. Med. 2011, 365, 2277-2286, doi:10.1056/NEJMoa1103944.

5. Basner, R.C. Continuous positive airway pressure for obstructive sleep apnea. N. Engl. J. Med. 2007, 356, 1751-1758, doi:356/17/1751 [pii]10.1056/NEJMct066953.

6. Randerath, W.; Bassetti, C.L.; Bonsignore, M.R.; Farre, R.; Ferini-Strambi, L.; Grote, L.; Hedner, J.; Kohler, M.; Martinez-Garcia, M.A.; Mihaicuta, S.; et al. Challenges and perspectives in obstructive sleep apnoea: Report by an ad hoc working group of the Sleep Disordered Breathing Group of the European Respiratory Society and the European Sleep Research Society. Eur. Respir. J. 2018, 52, doi:1702616 [pii]10.1183/13993003.02616-201713993003.02616-2017 [pii].

7. Penzel, T.; Schobel, C.; Fietze, I. New technology to assess sleep apnea: wearables, smartphones, and accessories. F1000Res 2018, 7, 413, doi:10.12688/f1000research.13010.1.

8. Young, T.; Evans, L.; Finn, L.; Palta, M. Estimation of the clinically diagnosed proportion of sleep apnea syndrome in middle-aged men and women. Sleep 1997, 20, 705-706, doi:10.1093/sleep/20.9.705.

9. $\quad$ Flemons, W.W.; Douglas, N.J.; Kuna, S.T.; Rodenstein, D.O.; Wheatley, J. Access to diagnosis and treatment of patients with suspected sleep apnea. Am. J. Respir. Crit. Care. Med. 2004, 169, 668-672, doi:10.1164/rccm.200308-1124PP169/6/668 [pii].

10. Drager, L.F. New Challenges for Sleep Apnea Research: Simple Diagnostic Tools, Biomarkers, New Treatments and Precision Medicine. Sleep Sci 2017, 10, 55-56, doi:10.5935/1984-0063.20170009.

11. Fitbit. How do I track my estimated oxygen variation in the Fitbit app? Available online: https://help.fitbit.com/articles/en_US/Help_article/1876.htm [Accessed July 08, 2021]

12. Garmin. Pulse Ox Frequently Asked Questions for Garmin Watches. Available online: https://support.garmin.com/en-US/?faq=SK2Y9a9aBp5D6n4sXmPBG7 [Accessed July 08, 2021] 
13. Huami. Amazfit X Bow to the Future. Available online: https://www.amazfit.com/en/amazfit-x.html [Accessed July 08, 2021].

14. Huawei. Huawei Watch 3. Available online: https:/consumer.huawei.com/en/wearables/watch-3/ [Accessed July 08, 2021].

15. Humon. Humon muscle oxygen sensor. Available online: https://humon.io/ [Accessed July 08, 2021].

16. Farzam, P.; Starkweather, Z.; Franceschini, M.A. Validation of a novel wearable, wireless technology to estimate oxygen levels and lactate threshold power in the exercising muscle. Physiological Reports 2018, 6, e13664, doi:https://doi.org/10.14814/phy2.13664.

17. Moxy. The Science Behind Moxy. Available http://www.moxymonitor.com/wp-content/themes/moxymonitor/documents/Moxy_Scientific Explanation_march2014.pdf [Accessed July 08, 2021].

18. Feldmann, A.; Schmitz, R.; Erlacher, D. Near-infrared spectroscopy-derived muscle oxygen saturation on a $0 \%$ to $100 \%$ scale: reliability and validity of the Moxy Monitor. J Biomed Opt 2019, 24, 1-11, doi:10.1117/1.JBO.24.11.115001.

19. Artinis. PortaMon. Available online: https://www.artinis.com/portamon [Accessed July 08, 2021].

20. Biofourmis AG. What does the Everion measure? Available online: https://support.biofourmis.com/hc/en-us/articles/213613165-What-does-the-Everion-measure- [Accessed July 08, 2021].

21. Villringer, A.; Chance, B. Non-invasive optical spectroscopy and imaging of human brain function. Trends Neurosci. 1997, 20, 435-442, doi:S0166-2236(97)01132-6 [pii].

22. Delpy, D.T.; Cope, M.; van der Zee, P.; Arridge, S.; Wray, S.; Wyatt, J. Estimation of optical pathlength through tissue from direct time of flight measurement. Phys. Med. Biol. 1988, 33, 1433-1442, doi:10.1088/0031-9155/33/12/008.

23. Scholkmann, F.; Kleiser, S.; Metz, A.J.; Zimmermann, R.; Mata Pavia, J.; Wolf, U.; Wolf, M. A review on continuous wave functional near-infrared spectroscopy and imaging instrumentation and methodology. Neuroimage 2014, 85 Pt 1, 6-27, doi:S1053-8119(13)00494-1 [pii]10.1016/j.neuroimage.2013.05.004.

24. Chan, E.D.; Chan, M.M. Pulse oximetry: understanding its basic principles facilitates appreciation of its limitations. Respir Med 2013, 107, 789-799, doi:10.1016/j.rmed.2013.02.004S0954-6111(13)00053-X [pii].

25. König, V.; Huch, R.; Huch, A. Reflectance Pulse Oximetry - Principles and Obstetric Application in the Zurich System. Journal of Clinical Monitoring and Computing 1998, 14, 403-412, doi:10.1023/a:1009983010772.

26. Nitzan, M.; Romem, A.; Koppel, R. Pulse oximetry: fundamentals and technology update. Med Devices (Auckl) 2014, 7, 231-239, doi:10.2147/MDER.S47319mder-7-231 [pii].

27. Menghini, L.; Cellini, N.; Goldstone, A.; Baker, F.C.; de Zambotti, M. A standardized framework for testing the performance of sleep-tracking technology: step-by-step guidelines and open-source code. Sleep 2021, 44, doi:zsaa170 [pii]10.1093/sleep/zsaa1705901094 [pii].

28. Zhang, Z.; Qi, M.; Hugli, G.; Khatami, R. The Challenges and Pitfalls of Detecting Sleep Hypopnea Using a Wearable Optical Sensor: Comparative Study. J. Med. Internet Res. 2021, 23, e24171, doi:10.2196/24171v23i7e24171 [pii].

29. Berry, R.B.; Kushida, C.A.; Kryger, M.H.; Soto-Calderon, H.; Staley, B.; Kuna, S.T. Respiratory event detection by a positive airway pressure device. Sleep 2012, 35, 361-367, doi:10.5665/sleep.1696.

30. Kim, D.E.; Hwangbo, Y.; Bae, J.H.; Yang, K.I. Accuracy of residual apnea-hypopnea index obtained using the continuous positive airway pressure device: application of new version 2.0 scoring rules for respiratory events during sleep. Sleep Breath. 2015, 19, 1335-1341, doi:10.1007/s11325-015-1257-010.1007/s11325-015-1257-0 [pii].

31. Stepnowsky, C.; Zamora, T.; Barker, R.; Liu, L.; Sarmiento, K. Accuracy of positive airway pressure device-measured apneas and hypopneas: role in treatment followup. Sleep Disord 2013, 2013, 314589, doi:10.1155/2013/314589.

32. Weaver, T.E.; Sawyer, A.M. Adherence to continuous positive airway pressure treatment for obstructive sleep apnoea: implications for future interventions. Indian J. Med. Res. 2010, 131, 245-258.

33. Wolk, R.; Shamsuzzaman, A.S.; Somers, V.K. Obesity, sleep apnea, and hypertension. Hypertension 2003, 42, 1067-1074, doi:10.1161/01.HYP.0000101686.98973.A301.HYP.0000101686.98973.A3 [pii].

34. Watzman, H.M.; Kurth, C.D.; Montenegro, L.M.; Rome, J.; Steven, J.M.; Nicolson, S.C. Arterial and venous contributions to near-infrared cerebral oximetry. Anesthesiology 2000, 93, 947-953, doi:10.1097/00000542-200010000-00012.

35. Franceschini, M.A.; Thaker, S.; Themelis, G.; Krishnamoorthy, K.K.; Bortfeld, H.; Diamond, S.G.; Boas, D.A.; Arvin, K.; Grant, P.E. Assessment of infant brain development with frequency-domain near-infrared spectroscopy. Pediatr. Res. 2007, 61, 546-551, doi:10.1203/pdr.0b013e318045be99.

36. Berry, R.B.; Brooks, R.; Gamaldo, C.E.; Harding, S.M.; Lloyd, R.M.; Marcus, C.L.; Vaughn, B.V. The AASM Manual for the Scoring of Sleep and Associated Events: Rules, Terminology and Technical Specifications; American Academy of Sleep Medicine: Darien, IL, 2017.

37. Fantini, S.; Sassaroli, A. Frequency-Domain Techniques for Cerebral and Functional Near-Infrared Spectroscopy. Front Neurosci 2020, 14, 300, doi:10.3389/fnins.2020.00300.

38. Fantini, S.; Franceschini, M.-A.; Maier, J.S.; Walker, S.A.; Barbieri, B.B.; Gratton, E. Frequency-domain multichannel optical detector for noninvasive tissue spectroscopy and oximetry. OPTICE 1995, 34, 32-42. 
39. Toronov, V.; Webb, A.; Choi, J.H.; Wolf, M.; Safonova, L.; Wolf, U.; Gratton, E. Study of local cerebral hemodynamics by frequency-domain near-infrared spectroscopy and correlation with simultaneously acquired functional magnetic resonance imaging. Opt Express 2001, 9, 417-427, doi:65396 [pii].

40. Fantini, S.; Franceschini, M.A.; Fishkin, J.B.; Barbieri, B.; Gratton, E. Quantitative determination of the absorption spectra of chromophores in strongly scattering media: a light-emitting-diode based technique. Appl. Opt. 1994, 33, 5204-5213, doi:42110 [pii].

41. Kleiser, S.; Nasseri, N.; Andresen, B.; Greisen, G.; Wolf, M. Comparison of tissue oximeters on a liquid phantom with adjustable optical properties. Biomed Opt Express 2016, 7, 2973-2992, doi:10.1364/BOE.7.002973262556 [pii].

42. Stankovic, M.R.; Maulik, D.; Rosenfeld, W.; Stubblefield, P.G.; Kofinas, A.D.; Drexler, S.; Nair, R.; Franceschini, M.A.; Hueber, D.; Gratton, E.; et al. Real-time optical imaging of experimental brain ischemia and hemorrhage in neonatal piglets. J. Perinat. Med. 1999, 27, 279-286, doi:10.1515/JPM.1999.039.

43. Fantini, S.; Franceschini, M.; Gratton, E.; Hueber, D.; Rosenfeld, W.; Maulik, D.; Stubblefield, P.; Stankovic, M. Non-invasive optical mapping of the piglet brain in real time. Opt Express 1999, 4, 308-314, doi:63389 [pii]10.1364/oe.4.000308.

44. Hallacoglu, B.; Sassaroli, A.; Wysocki, M.; Guerrero-Berroa, E.; Schnaider Beeri, M.; Haroutunian, V.; Shaul, M.; Rosenberg, I.H.; Troen, A.M.; Fantini, S. Absolute measurement of cerebral optical coefficients, hemoglobin concentration and oxygen saturation in old and young adults with near-infrared spectroscopy. J. Biomed. Opt. 2012, 17, 081406-081401, doi:10.1117/1.JBO.17.8.0814061306761 [pii].

45. Fantini, S.; Hueber, D.; Franceschini, M.A.; Gratton, E.; Rosenfeld, W.; Stubblefield, P.G.; Maulik, D.; Stankovic, M.R. Non-invasive optical monitoring of the newborn piglet brain using continuous-wave and frequency-domain spectroscopy. Phys. Med. Biol. 1999, 44, 1543-1563, doi:10.1088/0031-9155/44/6/308.

46. Kleiser, S.; Ostojic, D.; Andresen, B.; Nasseri, N.; Isler, H.; Scholkmann, F.; Karen, T.; Greisen, G.; Wolf, M. Comparison of tissue oximeters on a liquid phantom with adjustable optical properties: an extension. Biomed Opt Express 2018, 9, 86-101, doi:10.1364/BOE.9.000086306200 [pii].

47. Zhang, Z.; Schneider, M.; Laures, M.; Qi, M.; Khatami, R. The Comparisons of Cerebral Hemodynamics Induced by Obstructive Sleep Apnea with Arousal and Periodic Limb Movement with Arousal: A Pilot NIRS Study. Front Neurosci 2016, 10, 403, doi:10.3389/fnins.2016.00403.

48. Zhang, Z.; Khatami, R. Predominant endothelial vasomotor activity during human sleep: a near-infrared spectroscopy study. Eur. J. Neurosci. 2014, 40, 3396-3404, doi:10.1111/ejn.12702.

49. Cleveland, W.S.; Devlin, S.J. Locally Weighted Regression - an Approach to Regression-Analysis by Local Fitting. J. Am. Stat. Assoc. 1988, 83, 596-610, doi:Doi 10.2307/2289282.

50. Nakagawa, S.; Schielzeth, H. A general and simple method for obtaining R2 from generalized linear mixed-effects models. Methods in Ecology and Evolution 2013, 4, 133-142, doi:10.1111/j.2041-210x.2012.00261.x.

51. Xu, R. Measuring explained variation in linear mixed effects models. Stat. Med. 2003, 22, 3527-3541, doi:10.1002/sim.1572.

52. Kandel, G.; Aberman, A. Mixed venous oxygen saturation. Its role in the assessment of the critically ill patient. Arch. Intern. Med. 1983, 143, 1400-1402, doi:10.1001/archinte.143.7.1400.

53. Benni, P.B.; MacLeod, D.; Ikeda, K.; Lin, H.M. A validation method for near-infrared spectroscopy based tissue oximeters for cerebral and somatic tissue oxygen saturation measurements. J. Clin. Monit. Comput. 2018, 32, 269-284, doi:10.1007/s10877-017-0015-110.1007/s10877-017-0015-1 [pii].

54. Kreeger, R.N.; Ramamoorthy, C.; Nicolson, S.C.; Ames, W.A.; Hirsch, R.; Peng, L.F.; Glatz, A.C.; Hill, K.D.; Hoffman, J.; Tomasson, J.; et al. Evaluation of pediatric near-infrared cerebral oximeter for cardiac disease. Ann. Thorac. Surg. 2012, 94, 1527-1533, doi:10.1016/j.athoracsur.2012.05.096S0003-4975(12)01256-8 [pii].

55. Bickler, P.E.; Feiner, J.R.; Rollins, M.D. Factors affecting the performance of 5 cerebral oximeters during hypoxia in healthy volunteers. Anesth Analg 2013, 117, 813-823, doi:10.1213/ANE.0b013e318297d763ANE.0b013e318297d763 [pii].

56. Henson, L.C.; Calalang, C.; Temp, J.A.; Ward, D.S. Accuracy of a cerebral oximeter in healthy volunteers under conditions of isocapnic hypoxia. Anesthesiology 1998, 88, 58-65, doi:10.1097/00000542-199801000-00011.

57. Shah, N.; Trivedi, N.K.; Clack, S.L.; Shah, M.; Shah, P.P.; Barker, S. Impact of hypoxemia on the performance of cerebral oximeter in volunteer subjects. J. Neurosurg. Anesthesiol. 2000, 12, 201-209, doi:10.1097/00008506-200007000-00001.

58. Benni, P.B.; Chen, B.; Dykes, F.D.; Wagoner, S.F.; Heard, M.; Tanner, A.J.; Young, T.L.; Rais-Bahrami, K.; Rivera, O.; Short, B.L. Validation of the CAS neonatal NIRS system by monitoring vv-ECMO patients: preliminary results. Adv. Exp. Med. Biol. 2005, 566, 195-201, doi:10.1007/0-387-26206-7_27.

59. Rais-Bahrami, K.; Rivera, O.; Short, B.L. Validation of a noninvasive neonatal optical cerebral oximeter in veno-venous ECMO patients with a cephalad catheter. J. Perinatol. 2006, 26, 628-635, doi:7211573 [pii]10.1038/sj.jp.7211573.

60. Ikeda, K.; MacLeod, D.B.; Grocott, H.P.; Moretti, E.W.; Ames, W.; Vacchiano, C. The accuracy of a near-infrared spectroscopy cerebral oximetry device and its potential value for estimating jugular venous oxygen saturation. Anesth. Analg. 2014, 119, 1381-1392, doi:10.1213/ANE.0000000000000463.

61. Redford, D.; Paidy, S.; Kashif, F. Absolute and trend accuracy of a new regional oximeter in healthy volunteers during controlled hypoxia. Anesth. Analg. 2014, 119, 1315-1319, doi:10.1213/ANE.000000000000047400000539-201412000-00018 [pii]. 
62. Franceschini, M.A.; Boas, D.A.; Zourabian, A.; Diamond, S.G.; Nadgir, S.; Lin, D.W.; Moore, J.B.; Fantini, S. Near-infrared spiroximetry: noninvasive measurements of venous saturation in piglets and human subjects. J Appl Physiol (1985) 2002, 92, 372-384, doi:10.1152/jappl.2002.92.1.372.

63. MacDonald, M.J.; Tarnopolsky, M.A.; Green, H.J.; Hughson, R.L. Comparison of femoral blood gases and muscle near-infrared spectroscopy at exercise onset in humans. J Appl Physiol (1985) 1999, 86, 687-693, doi:10.1152/jappl.1999.86.2.687.

64. Imadojemu, V.A.; Gleeson, K.; Gray, K.S.; Sinoway, L.I.; Leuenberger, U.A. Obstructive apnea during sleep is associated with peripheral vasoconstriction. Am. J. Respir. Crit. Care. Med. 2002, 165, 61-66, doi:10.1164/ajrccm.165.1.2009062.

65. Kraiczi, H.; Hedner, J.; Peker, Y.; Carlson, J. Increased vasoconstrictor sensitivity in obstructive sleep apnea. J Appl Physiol (1985) 2000, 89, 493-498, doi:10.1152/jappl.2000.89.2.493.

66. Smith, R.P.; Veale, D.; Pepin, J.L.; Levy, P.A. Obstructive sleep apnoea and the autonomic nervous system. Sleep Med Rev 1998, 2, 69-92, doi:S1087079298900016 [pii].

67. Andreas, S.; Hajak, G.; von Breska, B.; Ruther, E.; Kreuzer, H. Changes in heart rate during obstructive sleep apnoea. Eur Respir J 1992, 5, 853-857.

68. Tolle, F.A.; Judy, W.V.; Yu, P.L.; Markand, O.N. Reduced stroke volume related to pleural pressure in obstructive sleep apnea. J Appl Physiol Respir Environ Exerc Physiol 1983, 55, 1718-1724, doi:10.1152/jappl.1983.55.6.1718.

69. Kirszenblat, R.; Edouard, P. Validation of the Withings ScanWatch as a Wrist-Worn Reflective Pulse Oximeter: Prospective Interventional Clinical Study. J Med Internet Res 2021, 23, e27503, doi:10.2196/27503.

70. Reichmuth, K.J.; Dopp, J.M.; Barczi, S.R.; Skatrud, J.B.; Wojdyla, P.; Hayes, D., Jr.; Morgan, B.J. Impaired vascular regulation in patients with obstructive sleep apnea: effects of continuous positive airway pressure treatment. Am. J. Respir. Crit. Care. Med. 2009, 180, 1143-1150, doi:10.1164/rccm.200903-0393OC200903-0393OC [pii].

71. Spicuzza, L.; Bernardi, L.; Balsamo, R.; Ciancio, N.; Polosa, R.; Di Maria, G. Effect of treatment with nasal continuous positive airway pressure on ventilatory response to hypoxia and hypercapnia in patients with sleep apnea syndrome. Chest 2006, 130, 774-779, doi:S0012-3692(15)52790-6 [pii]10.1378/chest.130.3.774. 